This article was downloaded by: [B-on Consortium - 2007]

On: 25 August 2009

Access details: Access Details: [subscription number 908038079]

Publisher Taylor \& Francis

Informa Ltd Registered in England and Wales Registered Number: 1072954 Registered office: Mortimer House, 37-41 Mortimer Street, London W1T 3JH, UK

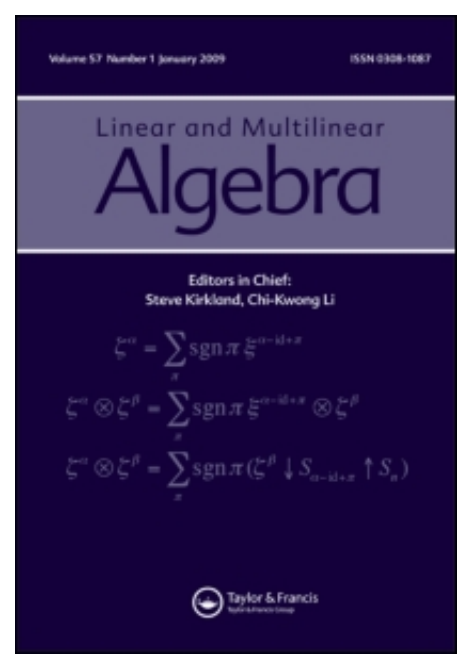

\title{
Linear and Multilinear Algebra
}

Publication details, including instructions for authors and subscription information:

http://www.informaworld.com/smpp/title content=t713644116

\section{The inverse eigenvalue problem for Hermitian matrices whose graphs are}

\section{cycles}

Rosário Fernandes a; C.M. da Fonseca ${ }^{b}$

a Departamento de Matemática, Universidade Nova de Lisboa, Caparica, Portugal ${ }^{\text {b }}$ Departamento de Matemática, Universidade de Coimbra, Coimbra, Portugal

First Published on: 15 December 2008

To cite this Article Fernandes, Rosário and Fonseca, C.M. da(2008)'The inverse eigenvalue problem for Hermitian matrices whose graphs are cycles',Linear and Multilinear Algebra,99999:1,

To link to this Article: DOI: $10.1080 / 03081080802187870$

URL: http://dx.doi.org/10.1080/03081080802187870

\section{PLEASE SCROLL DOWN FOR ARTICLE}

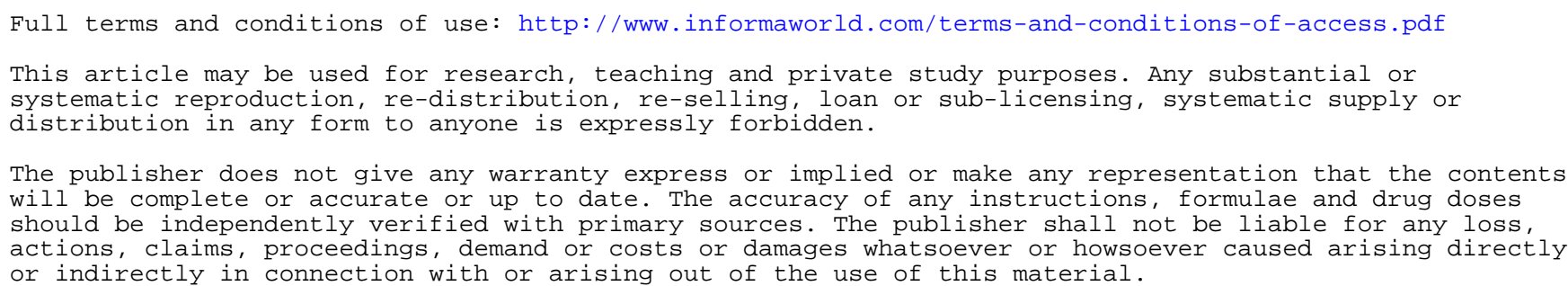




\title{
The inverse eigenvalue problem for Hermitian matrices whose graphs are cycles
}

\author{
Rosário Fernandes ${ }^{\mathrm{a}}$ and C.M. da Fonseca ${ }^{\mathrm{b} *}$ \\ ${ }^{a}$ Departamento de Matemática, Universidade Nova de Lisboa, Caparica, Portugal; \\ ${ }^{b}$ Departamento de Matemática, Universidade de Coimbra, Coimbra, Portugal
}

Communicated by R.P. Bapat

(Received 24 August 2007; final version received 4 May 2008)

In 1979, Ferguson characterized the periodic Jacobi matrices with given eigenvalues and showed how to use the Lanzcos Algorithm to construct each such matrix. This article provides general characterizations and constructions for the complex analogue of periodic Jacobi matrices. As a consequence of the main procedure, we prove that the multiplicity of an eigenvalue of a periodic Jacobi matrix is at most 2 .

Keywords: inverse eigenvalue problem; periodic Jacobi matrix; eigenvalues; multiplicities; graphs; cycle

AMS subject classification: $15 \mathrm{~A} 18$

\section{Introduction}

A periodic Jacobi matrix is a real symmetric matrix of the form

$$
L=\left(\begin{array}{cccc}
a_{1} & b_{1} & & b_{n} \\
b_{1} & \ddots & \ddots & \\
& \ddots & \ddots & b_{n-1} \\
b_{n} & & b_{n-1} & a_{n}
\end{array}\right),
$$

where $b_{i}>0$, for $i=1, \ldots, n$, and all the non-mentioned entries are zero.

Extensive attention has been paid in the literature to the theory of periodic Jacobi matrices (cf. $[1,2,3,7,10])$. Many problems on the spectra of periodic Jacobi matrices arise in a remarkable variety of applications, in pure and applied mathematics.

Ferguson [3] presented an algorithm for calculating $L$ from some given spectral data, based on the Lanczos algorithm as treated by Boley and Golub [2], using a discrete version of Floquet theory. It is a typical inverse eigenvalue problem, a problem concerning the reconstruction of a matrix from prescribed spectral data.

Let $J$ denote the Jacobi matrix obtained by deleting from the periodic matrix (1) the last row and column, with characteristic polynomial

$$
\omega_{J}(\lambda)=\operatorname{det}(\lambda I-J)=\left(\lambda-\mu_{1}\right) \cdots\left(\lambda-\mu_{n-1}\right) .
$$

*Corresponding author. Email: cmf@mat.uc.pt 
We assume a Jacobi matrix to be any real, symmetric tridiagonal matrix whose next diagonal entries are positive [4-6]. Let $\mu_{1}, \ldots, \mu_{n-1}$ be the eigenvalues of $J, u_{1}, \ldots, u_{n-1}$ be the first components of a set $Y_{1}, \ldots, Y_{n-1}$ of real orthonormal eigenvectors of $J$ associated with eigenvalues $\mu_{1}, \ldots, \mu_{n-1}$, respectively.

Definition 1.1 [3] The Floquet multipliers $\rho_{1}, \ldots, \rho_{n-1}$ of $L$ corresponding to $\mu_{1}, \ldots, \mu_{n-1}$ are the numbers defined by the relation

$$
b_{1} \cdots b_{n}=-\rho_{j} \omega_{J}^{\prime}\left(\mu_{j}\right) b_{n}^{2} u_{j}^{2}, \quad j=1, \ldots, n-1 .
$$

Ferguson [3] showed that for given real numbers $\Lambda, B(>0), \mu_{1}>\cdots>\mu_{n-1}$ and $\rho_{1}, \ldots, \rho_{n-1}$ such that

$$
\rho_{j} \omega_{J}^{\prime}\left(\mu_{j}\right)<0, \quad \text { for } j=1, \ldots, n-1,
$$

there exists a unique periodic Jacobi matrix $L(1)$ such that

$$
a_{1}+a_{2}+\cdots+a_{n}=\Lambda \text { and } b_{1} \cdots b_{n}=B,
$$

where $\mu_{i}, \quad i=1, \ldots, n-1$, are the eigenvalues of $J$ and the $\rho_{j}$ are the Floquet multipliers of $L$.

Ferguson also based his analysis on the partial characterization of periodic Jacobi matrices by van Moerbeke who in [10] had given an analogue of Floquet theory for a different periodic Jacobi matrix.

Later, Andrea and Berry [1] presented some algorithms based on a continued fraction expansion for solving the inverse eigenvalue problem for periodic Jacobi matrices.

In this work, we will see a periodic Jacobi complex matrix as the adjacency matrix of weighted cycle. After an introduction with some results on the characteristic polynomial of a weighted graph, we establish an algorithm for the construction of general periodic Jacobi matrices with a given spectra. This procedure is based on Ferguson's algorithm, but it is more general. A final corollary states that the multiplicity of an eigenvalue of a periodic Jacobi matrix is at most 2.

\section{The characteristic polynomial of a weighted graph}

A graph $G=(\mathcal{V}, \mathcal{E})$ consists of a finite set $\mathcal{V}=\mathcal{V}(G)$ whose members are called vertices, and a set $\mathcal{E}=\mathcal{E}(G)$ of 2-subsets of $\mathcal{V}$, whose members are called edges. By a digraph $D=(\mathcal{V}, \mathcal{A})$ we mean the same finite set $\mathcal{V}$, and a subset $\mathcal{A}=\mathcal{A}(D)$ of $\mathcal{V} \times \mathcal{V}$, whose members are called arcs. Note that an arc is an ordered pair $(i, j)$, whereas an edge of a graph is also a pair but is unorder. We write in both contexts $i \sim j$.

A directed path from $i_{1}$ to $i_{r}, P_{i_{1}, i_{r}}$, in the digraph $D$ is a sequence of distinct vertices $\left(i_{1}, i_{2}, \ldots, i_{r-1}, i_{r}\right)$ such that each arc $\left(i_{1}, i_{2}\right), \ldots,\left(i_{r-1}, i_{r}\right)$ is in $\mathcal{A}(D)$. The length of $P_{i_{1}, i_{r}}, \ell\left(P_{i_{1}, i_{r}}\right)$, is $r-1$. If to the path $P_{i_{1}, i_{r}}$ we add the arc $\left(i_{r}, i_{1}\right)$, then we have a directed cycle $\left(i_{1}, i_{2}, \ldots, i_{r}, i_{1}\right)$ (of length $r$ ). Analogously, the path from $i_{1}$ to $i_{r}$ in the simple graph $G$ is a sequence of distinct vertices $\left(i_{1}, i_{2}, \ldots, i_{r-1}, i_{r}\right)$ such that each edge $\left\{i_{1}, i_{2}\right\}, \ldots,\left\{i_{r-1}, i_{r}\right\}$ is in $\mathcal{E}(G)$. If to this path we add the edge $\left\{i_{r}, i_{1}\right\}$, then we have a cycle $\left(i_{1}, i_{2}, \ldots, i_{r}, i_{1}\right)$ of length $r$. If any two vertices can be joined by a path we say that the graph is connected. A forest is a graph without cycles and a tree is a connected forest. 
Given an $\operatorname{arc} e=(i, j)$ of $D, D \backslash e$ is obtained by deleting $e$ but not the vertices $i$ or $j$; the sub-digraph $D \backslash X$, where $X$ is a subset of vertices of $D$, is obtained from $D$ deleting the vertices $X$ and all arcs incident with vertices of $X$.

Let $A=\left(a_{i j}\right)$ be an $n \times n$ matrix. The graph of $A, G(A)$, is the pair $(\mathcal{V}, \mathcal{E})$, where $\mathcal{V}=\{1, \ldots, n\}$ and $(i, j), i \neq j$, is an edge in $\mathcal{E}$ if and only if $a_{i j} \neq 0$ or $a_{j i} \neq 0$. Analogously, the digraph $D(A)=(\mathcal{V}, \mathcal{A})$ of $A=\left(a_{i j}\right)$ contains the arc $(i, j) \in \mathcal{A}$ if and only if $a_{i j} \neq 0$. The matrix $A$ can be viewed as a weighted adjacency matrix of the digraph $D(A)$, with loops (arcs of the type $(i, i))$ allowed on the vertices.

We denote by $A(X)$, where $X$ is a subset of vertices of the graph or digraph of $A$, the submatrix obtained by deleting from $A$ the rows and columns labelled by $X$.

We have a general formula for the determinant:

Theorem 2.1 [9] Given an $n \times n$ matrix $A=\left(a_{i j}\right)$ and $r \in\{1, \ldots, n\}$, let us assume that $\left\{C_{1}, \ldots, C_{m}\right\}$ is the set of all directed cycles in $D(A)=D$ containing the vertex $r$, with $\ell_{j}=\ell\left(C_{j}\right)$. Then

$$
\operatorname{det} A=\sum_{k=1}^{m}(-1)^{\ell_{k}+1} \operatorname{det} A\left(\mathcal{V}\left(C_{k}\right)\right) \prod_{(i, j) \in \mathcal{A}\left(C_{k}\right)} a_{i j},
$$

where $C_{k}=\left(\mathcal{V}\left(C_{k}\right), \mathcal{A}\left(C_{k}\right)\right)$ and $\operatorname{det} A\left(\mathcal{V}\left(C_{k}\right)\right)=1$ if $C_{k}$ contains all vertices of $D$.

The set of cycles includes the cycles of one arc (a loop), the cycles with two $\operatorname{arcs},(i, j, i)$, if $a_{i j} \neq 0$ and $a_{j i} \neq 0$, and so on.

Suppose now $A$ is Hermitian. Theorem 2.1 provides a general formula for the characteristic polynomial of $A, \varphi_{A}(\lambda)=\operatorname{det}(\lambda I-A)$.

Corollary 2.2 Given an $n \times n$ Hermitian matrix $A=\left(a_{i j}\right)$ and $i \in\{1, \ldots, n\}$, let us assume that $\left\{C_{1}, \ldots, C_{m}\right\}$ is the set of all cycles in $G(A)=G$ containing the vertex $i$, with $C_{k}=\left(k_{1}, \ldots, k_{\ell_{k}}, k_{1}\right), k=1, \ldots, m$. Then

$$
\begin{aligned}
\varphi_{A}(\lambda)= & \left(\lambda-a_{i i}\right) \varphi_{A(i)}(\lambda)-\sum_{j \sim i}\left|a_{i j}\right|^{2} \varphi_{A(i, j)}(\lambda) \\
& -2 \sum_{k=1}^{m} \operatorname{Re}\left(a_{k_{1} k_{2}} \cdots a_{k_{\ell_{k}-1} k_{\ell_{k}}} \bar{a}_{k_{\ell_{k}} k_{1}}\right) \varphi_{A\left(\mathcal{V}\left(C_{k}\right)\right)}(\lambda),
\end{aligned}
$$

If the graph of $A$ contains only one cycle, then we conclude the following:

Corollary 2.3 Given an $n \times n$ Hermitian matrix $A=\left(a_{i j}\right)$ whose graph $G$ has only one cycle, say $C=(1, \ldots, \ell, 1)$, let $i \in\{1, \ldots, \ell\}$ be a vertex of $C$. Then

$$
\varphi_{A}(\lambda)=\left(\lambda-a_{i i}\right) \varphi_{A(i)}(\lambda)-\sum_{j \sim i}\left|a_{i j}\right|^{2} \varphi_{A(i, j)}(\lambda)-2 \operatorname{Re}\left(a_{12} \cdots a_{\ell-1, \ell} \bar{a}_{\ell, 1}\right) \varphi_{A(\mathcal{V}(C))}(\lambda) .
$$

Corollary 2.4 Given an $n \times n$ Hermitian matrix $A=\left(a_{i j}\right)$ whose graph $G$ is a cycle, say $(1, \ldots, n, 1)$, and $i \in\{1, \ldots, n\}$, the characteristic polynomial of $A$ is

$$
\begin{aligned}
\varphi_{A}(\lambda) & =\left(\lambda-a_{i i}\right) \varphi_{A(i)}(\lambda)-\left|a_{i-1, i}\right|^{2} \varphi_{A(i-1, i)}(\lambda) \\
& -\left|a_{i, i+1}\right|^{2} \varphi_{A(i, i+1)}(\lambda)-2 \operatorname{Re}\left(a_{12} \cdots a_{n-1, n} \bar{a}_{n, 1}\right) .
\end{aligned}
$$


Corollary 2.5 Given an $n \times n$ Hermitian matrix $A=\left(a_{i j}\right)$ whose graph $G$ is a path, say $(1, \ldots, n)$, and $i \in\{1, \ldots, n\}$, the characteristic polynomial of $A$ is

$$
\varphi_{A}(\lambda)=\left(\lambda-a_{i i}\right) \varphi_{A(i)}(\lambda)-\sum_{j \sim i}\left|a_{i j}\right|^{2} \varphi_{A(i, j)}(\lambda)
$$

For a Hermitian matrix $A=\left(a_{i j}\right)$, let us denote the corresponding symmetric matrix of the modulus of $A$ by $A^{+}=\left(\left|a_{i j}\right|\right)$.

Corollary 2.6 Given an $n \times n$ Hermitian matrix $A=\left(a_{i j}\right)$ whose graph $G$ is a path, say $(1, \ldots, n)$, and $i \in\{1, \ldots, n\}$, then

$$
\varphi_{A}(\lambda)=\varphi_{A^{+}}(\lambda)
$$

\section{Inverse eigenvalue problem}

The main aim of an inverse eigenvalue problem is to construct a matrix that maintains a certain specific structure as well as some given spectral property. Given distinct real numbers $\mu_{1}, \ldots, \mu_{n-1}$ and non-zero real numbers, $u_{1}, \ldots, u_{n-1}$, whose squares sum is 1, Ferguson [3] used Lanzcos algorithm to get a Jacobi matrix

$$
J=\left(\begin{array}{cccc}
a_{1} & b_{1} & & \\
b_{1} & \ddots & \ddots & \\
& \ddots & \ddots & b_{n-2} \\
& & b_{n-2} & a_{n-1}
\end{array}\right),
$$

such that $u_{1}, \ldots, u_{n-1}$ are the first components of a set $Y_{1}, \ldots, Y_{n-1}$ of real orthonormal eigenvectors of $J$ associated with eigenvalues $\mu_{1}, \ldots, \mu_{n-1}$, based on some relationships between the eigenvalues and eigenvectors of $J$ :

Algorithm 1 [3]

1. Set:

1.1: $b_{0}=1$;

1.2: $u_{0, j}=0$, for $j=1,2, \ldots, k$;

1.3: $u_{1, j}=u_{j}$, for $j=1,2, \ldots, k$.

2. Iteration $i=1,2, \ldots, k-1$ :

2.1: $a_{i}=\sum_{\ell=1}^{k} \mu_{\ell} u_{i, \ell}^{2}$;

2.2: $b_{i}=\sqrt{\sum_{\ell=1}^{k}\left(\left(\mu_{\ell}-a_{i}\right) u_{i, \ell}-b_{i-1} u_{i-1, \ell}\right)^{2}}$;

2.3: $u_{i+1, j}=\left(\left(\mu_{j}-a_{i}\right) u_{i, j}-b_{i-1} u_{i-1, j}\right) / b_{i}$, for $j=1,2, \ldots, k$.

3. $a_{k}=\sum_{\ell=1}^{k} \mu_{\ell} u_{k, \ell}^{2}$.

In [8], Leal Duarte generalized this construction to any Hermitian matrix whose graph is a tree. Ferguson also treated an inverse eigenvalue problem for periodic Jacobi matrices, which we generalize here. 
The matrix

$$
L_{\rho}=\left(\begin{array}{cccc}
a_{1} & b_{1} & & \frac{1}{\rho} b_{n} \\
b_{1} & \ddots & \ddots & \\
& \ddots & \ddots & b_{n-1} \\
\rho b_{n} & & b_{n-1} & a_{n}
\end{array}\right),
$$

where $\rho \neq 0$, has some interesting spectral properties:

THEOREM 3.1 [3] The characteristic polynomial of $L_{\rho}$ admits the representation

$$
\operatorname{det}\left(\lambda I-L_{\rho}\right)=b_{1} \cdots b_{n}\left(\Delta(\lambda)-\left(\rho+\frac{1}{\rho}\right)\right)
$$

where $\Delta(\lambda)$, called the discriminant of $L_{\rho}$, is independent of $\rho$. The Floquet multipliers $\rho_{1}, \ldots, \rho_{n-1}$ of $L$ corresponding to the eigenvalues $\mu_{1}, \ldots, \mu_{n-1}$ of $J$ satisfy the relation

$$
(-1)^{j} \Delta\left(\mu_{j}\right)=(-1)^{j}\left(\rho_{j}+\frac{1}{\rho_{j}}\right) \geq 2, \quad j=1, \ldots, n-1 .
$$

Furthermore, the eigenvalues $\lambda_{1}, \ldots, \lambda_{n}$ of $L$, which are the roots of $\Delta(\lambda)=2$, are real and can be ordered so that

$$
\lambda_{1}>\lambda_{2} \geq \lambda_{3}>\lambda_{4} \geq \lambda_{5}>\cdots
$$

Now, suppose that $A$ is a Hermitian matrix whose graph is exactly the cycle $C=(1, \ldots, n, 1)$, i.e.

$$
A=\left(\begin{array}{cccc}
a_{1} & b_{1} & & b_{n} \\
\overline{b_{1}} & \ddots & \ddots & \\
& \ddots & \ddots & b_{n-1} \\
\bar{b}_{n} & & \bar{b}_{n-1} & a_{n}
\end{array}\right),
$$

where $a_{\ell}$ 's are real numbers and $b_{\ell}$ 's are non-zero complex numbers.

Recall that the main characterization result by Ferguson states:

Theorem 3.2 [3] There exists a periodic Jacobi matrix (1) with eigenvalues $\lambda_{1}, \ldots, \lambda_{n}$ if and only if the real numbers $\lambda_{1}, \ldots, \lambda_{n}$ can be rearranged such that

$$
\lambda_{1}>\lambda_{2} \geq \lambda_{3}>\lambda_{4} \geq \cdots \text {. }
$$

Let us now consider the case when

$$
\lambda_{1} \geq \lambda_{2}>\lambda_{3} \geq \lambda_{4}>\cdots .
$$

Set

$$
\Lambda=\lambda_{1}+\lambda_{2}+\cdots+\lambda_{n},
$$


and define real numbers $\mu_{1}>\mu_{2}>\cdots>\mu_{n-1}$ and $B(>0)$ such that

$$
(-1)^{j} \Delta\left(\mu_{j}\right) \geq 2, \quad \text { for } j=1,2, \ldots, n-1,
$$

and

$$
\lambda_{1} \geq \mu_{1} \geq \lambda_{2} \geq \mu_{2} \geq \cdots
$$

where

$$
\Delta(\lambda)=-2+\frac{1}{B}\left(\lambda-\lambda_{1}\right) \cdots\left(\lambda-\lambda_{n}\right) .
$$

Considering $\rho_{1}, \ldots, \rho_{n-1}$ such that

$$
\Delta\left(\mu_{j}\right)=-\rho_{j}-\frac{1}{\rho_{j}}, \quad \text { for } j=1,2, \ldots, n-1,
$$

with $\omega_{J}(\lambda)=\left(\mu-\mu_{1}\right) \cdots\left(\mu-\mu_{n-1}\right)$, we establish the following algorithm:

\section{Algorithm 2}

1. Set:

1.1: $b_{n}=\sqrt{\sum_{\ell=1}^{n-1}\left(B / \rho_{\ell} \omega_{J}^{\prime}\left(\mu_{\ell}\right)\right)}$;

1.2: $u_{\ell}=1 / b_{n} \sqrt{\left(B / \rho_{\ell} \omega_{J}^{\prime}(\ell)\right)}$, for $\ell=1,2, \ldots, n-1$.

2. Use Algorithm 1 to contruct a Jacobi matrix (6).

3. Set:

$$
\text { 3.1: } b_{n-1}=B /\left(b_{1} b_{2} \ldots b_{n-2} b_{n}\right) \text {; }
$$

3.2: $a_{n}=\Lambda-\left(a_{1}+a_{2}+\cdots+a_{n-1}\right)$.

We are now able to state a more general result containing the Theorem 3.2.

THeOREM 3.3 Let $\lambda_{1}, \ldots, \lambda_{n}$ be real numbers. If $A$ is a Hermitian matrix (10) with eigenvalues $\lambda_{1}, \ldots, \lambda_{n}$, then $\lambda_{1}, \ldots, \lambda_{n}$ can be ordered as

$$
\lambda_{1} \geq \lambda_{2}>\lambda_{3} \geq \lambda_{4}>\cdots, \quad \text { if } \operatorname{Re}\left(b_{1} \cdots b_{n-1} \bar{b}_{n}\right)<0
$$

or

$$
\lambda_{1}>\lambda_{2} \geq \lambda_{3}>\lambda_{4} \geq \cdots, \quad \text { if } \operatorname{Re}\left(b_{1} \cdots b_{n-1} \bar{b}_{n}\right)>0 .
$$

Conversely, if

$$
\lambda_{1} \geq \lambda_{2}>\lambda_{3} \geq \lambda_{4}>\cdots
$$

then $\lambda_{1}, \ldots, \lambda_{n}$ are the eigenvalues of a symmetric matrix as in (1), with $b_{1} \cdots b_{n}<0$. Similarly, if

$$
\lambda_{1}>\lambda_{2} \geq \lambda_{3}>\lambda_{4} \geq \cdots,
$$

$\lambda_{1}, \ldots, \lambda_{n}$ are the original eigenvalues of some periodic Jacobi matrix as in (1).

Proof We start with the necessity. By Corollary 2.4, the characteristic polynomial of $A$ is $\varphi_{A}(\lambda)=\left(\lambda-a_{1}\right) \varphi_{A(1)}(\lambda)-\left|b_{1}\right|^{2} \varphi_{A(1,2)}(\lambda)-\left|b_{n}\right|^{2} \varphi_{A(1, n)}(\lambda)-2 \operatorname{Re}\left(b_{1} \cdots b_{n-1} \bar{b}_{n}\right)$. 
If one considers the symmetric matrix

$$
A^{+}=\left(\begin{array}{cccc}
a_{1} & \left|b_{1}\right| & & \left|b_{n}\right| \\
\left|b_{1}\right| & \ddots & \ddots & \\
& \ddots & \ddots & \left|b_{n-1}\right| \\
\left|b_{n}\right| & & \left|b_{n-1}\right| & a_{n}
\end{array}\right),
$$

then, again by Corollary 2.4, the characteristic polynomial of $A^{+}$is

$$
\varphi_{A^{+}}(\lambda)=\left(\lambda-a_{1}\right) \varphi_{A^{+}(1)}(\lambda)-\left|b_{1}\right|^{2} \varphi_{A^{+}(1,2)}(\lambda)-\left|b_{n}\right|^{2} \varphi_{A^{+}(1, n)}(\lambda)-2\left|b_{1} \cdots b_{n}\right| .
$$

Hence by Corollary 2.6,

$$
\varphi_{A^{+}(1)}(\lambda)=\varphi_{A(1)}(\lambda), \quad \varphi_{A^{+}(1,2)}(\lambda)=\varphi_{A(1,2)}(\lambda), \quad \varphi_{A^{+}(1, n)}(\lambda)=\varphi_{A(1, n)}(\lambda),
$$

and then

$$
\varphi_{A}(\lambda)=\varphi_{A^{+}}(\lambda)+2\left|b_{1} \cdots b_{n}\right|-2 \operatorname{Re}\left(b_{1} \cdots b_{n-1} \bar{b}_{n}\right) .
$$

If

$$
\Delta_{A^{+}}(\lambda)=\left|b_{1} \cdots b_{n}\right|^{-1} \varphi_{A^{+}}(\lambda)+2
$$

(the so-called discriminant of $A^{+}$), then

$$
\varphi_{A}(\lambda)=\left|b_{1} \cdots b_{n}\right|\left(\Delta_{A^{+}}(\lambda)-2 \frac{\operatorname{Re}\left(b_{1} \cdots b_{n-1} n\right)}{\left|b_{1} \cdots b_{n}\right|}\right) .
$$

Since $\mid \operatorname{Re}\left(b_{1} \cdots b_{n-1}|\leq| b_{1} \cdots b_{n-1} \mid\right.$, the eigenvalues of $A$, which are the roots of

$$
\Delta_{A^{+}}(\lambda)=2 \frac{\operatorname{Re}\left(b_{1} \cdots b_{n-1} \bar{b}_{n}\right)}{\left|b_{1} \cdots b_{n}\right|},
$$

verify

$$
-2 \leq 2 \frac{\operatorname{Re}\left(b_{1} \cdots b_{n-1} \bar{b}_{n}\right)}{\left|b_{1} \cdots b_{n}\right|} \leq 2 .
$$

If $\mu_{1}>\cdots>\mu_{n-1}$ are the eigenvalues of the Jacobi matrix obtained by deleting from $A^{+}$the last row and column, then, using Theorem 3.1,

$$
(-1)^{j} \Delta_{A^{+}}\left(\mu_{j}\right) \geq 2, \quad j=1, \ldots, n-1 .
$$

Consequently, the eigenvalues of $A$ are real and can be ordered so that

$$
\lambda_{1} \geq \lambda_{2}>\lambda_{3} \geq \lambda_{4}>\cdots, \quad \text { if } \operatorname{Re}\left(b_{1} \cdots b_{n-1} \bar{b}_{n}\right)<0
$$

or

$$
\lambda_{1}>\lambda_{2} \geq \lambda_{3}>\lambda_{4} \geq \cdots, \quad \text { if } \operatorname{Re}\left(b_{1} \cdots b_{n-1} \bar{b}_{n}\right)>0,
$$

because the coefficient $\left|b_{1} \cdots b_{n}\right|^{-1}$ of $\lambda^{n}$ in $\Delta_{A^{+}}(\lambda)$ is positive. 
Conversely, from Theorem 3.2, if the real numbers $\lambda_{1}, \ldots, \lambda_{n}$ verify (12), then we can find such matrix. On the other hand, if those real numbers satisfy condition (11), according to Algorithm 2,

$$
T_{1}=\left(\begin{array}{ccccc}
a_{1} & b_{1} & & & b_{n} \\
b_{1} & & \ddots & & \\
& \ddots & \ddots & b_{n-2} & \\
& & b_{n-2} & a_{n-1} & -b_{n-1} \\
b_{n} & & & -b_{n-1} & a_{n}
\end{array}\right)
$$

is the desired matrix. In fact, let

$$
T_{\rho}=\left(\begin{array}{ccccc}
a_{1} & b_{1} & & & \frac{1}{\rho} b_{n} \\
b_{1} & \ddots & \ddots & & \\
& \ddots & \ddots & b_{n-2} & \\
& & b_{n-2} & a_{n-1} & -b_{n-1} \\
\rho b_{n} & & & -b_{n-1} & a_{n}
\end{array}\right), \quad \text { with } \rho \neq 0 .
$$

Since $u_{\ell}=\left(1 / b_{n}\right) \sqrt{\left(B / \rho_{\ell} \omega_{J}^{\prime}(\ell)\right)}$ and $B=b_{1} \cdots b_{n}$, we have

$$
b_{1} \cdots b_{n}=\rho_{\ell} \omega_{J}^{\prime}\left(\mu_{\ell}\right) b_{n}^{2} u_{\ell}^{2}, \quad \text { for } \ell=1,2, \ldots, n-1 .
$$

If $Y_{1}, \ldots, Y_{n-1}$ are the orthonormal eigenvectors of $J$, corresponding to its eigenvalues $\mu_{1}, \ldots, \mu_{n-1}$, obtained using Algorithm 1, then let $u_{i, \ell}$ denote the $i$-th component of $Y_{\ell}$. From the last equality and from the identity

$$
b_{1} \cdots b_{n-2}=\omega_{J}^{\prime}\left(\mu_{j}\right) u_{1, j} u_{n-1, j}, \quad \text { for } j=1, \ldots, n-1,
$$

we get

$$
\rho_{\ell} b_{n} u_{1, \ell}-b_{n-1} u_{n-1, \ell}=0, \quad \text { with } \ell=1, \ldots, n-1 \text {. }
$$

Consequently

$$
T_{\rho_{\ell}}\left(\begin{array}{c}
Y_{\ell} \\
0
\end{array}\right)=\mu_{\ell}\left(\begin{array}{c}
Y_{\ell} \\
0
\end{array}\right), \quad \text { for } \ell=1, \ldots, n-1 .
$$

Therefore, $\mu_{\ell}$ is an eigenvalue of $T_{\rho_{\ell}}$, for $\ell=1,2, \ldots, n-1$. Using elementary properties of determinants, it is easy to see that $(\mathrm{d} / \mathrm{d} \rho) \operatorname{det}\left(\lambda I-T_{\rho}\right)=b_{1} \cdots b_{n}\left(1-\left(1 / \rho^{2}\right)\right)$. When both sides are integrated with respect to $\rho$, we obtain

$$
\operatorname{det}\left(\lambda I-T_{\rho}\right)=b_{1} \cdots b_{n}\left(\Delta_{T}(\lambda)+\left(\rho+\frac{1}{\rho}\right)\right) .
$$

Then

$$
\Delta_{T}\left(\mu_{\ell}\right)=-\rho_{\ell}-\frac{1}{\rho_{\ell}}, \quad \text { for } \ell=1,2, \ldots, n-1 .
$$


Note that

$$
\Delta_{T}(\lambda)=\frac{1}{b_{1} \cdots b_{n}}\left(\lambda^{n}-\Lambda \lambda^{n-1}+\cdots\right)
$$

and thus the coefficients of $\lambda^{n}$ and $\lambda^{n-1}$ in $\Delta_{T}(\lambda)$ and $\Delta(\lambda)$, respectively, are the same. Hence, $\Delta_{T}(\lambda)-\Delta(\lambda)$ is a polynomial of degree $\leq n-2$. But $\Delta_{T}(\mu \ell)-\Delta\left(\mu_{\ell}\right)=0$, for $\ell=1,2, \ldots, n-1$, which means that $\Delta_{T}(\lambda)-\Delta(\lambda)$ has $n-1$ distinct roots. Therefore, $\Delta_{T}=\Delta$, and so $\lambda_{1}, \ldots, \lambda_{n}$ are the eigenvalues of $T_{1}$.

Example 3.1 Given the numbers 6, 3, 1, we want to find a Hermitian matrix (10) whose eigenvalues are

$$
\lambda_{1}=6=\lambda_{2}>\lambda_{3}=3=\lambda_{4}>\lambda_{5}=1 .
$$

We can get with $\Lambda=19, B=1, \mu_{1}=6>\mu_{2}=5>\mu_{3}=3, \mu_{4}=2$ and applying Algorithm 2, the matrix

$$
\left(\begin{array}{ccccc}
4 & \sqrt{2} & 0 & 0 & \sqrt{2-\sqrt{3}} \\
\sqrt{2} & 4+\frac{\sqrt{3}}{2} & \frac{1}{2} & 0 & 0 \\
0 & \frac{1}{2} & 4-\frac{\sqrt{3}}{2} & \sqrt{2} & 0 \\
0 & 0 & \sqrt{2} & 4 & -\sqrt{2+\sqrt{3}} \\
\sqrt{2-\sqrt{3}} & 0 & 0 & -\sqrt{2+\sqrt{3}} & 3
\end{array}\right)
$$

whose eigenvalues are given in (13).

Corollary 3.4 Any eigenvalue of a Hermitian matrix of the form (10) has at most multiplicity 2.

\section{Acknowledgements}

The authors are grateful to the referee whose constructive comments and suggestions helped to improve this article. This work was supported by CMUC-Centro de Matemática da Universidade de Coimbra.

\section{References}

[1] S.A. Andrea and T.G. Berry, Continued fractions and periodic Jacobi matrices, Lin. Alg. Appl. 161 (1992), pp. 117-134.

[2] D. Boley and G.H. Golub, A modified method for reconstructing periodic Jacobi matrices, Math. Comp. 42 (1984), pp. 143-150.

[3] W.E. Ferguson, The construction of Jacobi and periodic Jacobi matrices with prescribed spectra, Math. Comp. 35 (1980), pp. 1203-1220.

[4] L.J. Gray and D.G. Wilson, Construction of a Jacobi matrix from spectral data, Lin. Alg. Appl. 14 (1976), pp. 131-134.

[5] O.L. Hald, Inverse eigenvalue problems for Jacobi matrices, Lin. Alg. Appl. 14 (1976), pp. 63-85.

[6] H. Hochstadt, On the construction of a Jacobi matrix from spectral data, Lin. Alg. Appl. 8 (1974), pp. 435-446. 
[7] E. Korotyaev and I.V. Krasovsky, Spectral estimates for periodic Jacobi matrices, Comm. Math. Phys 234 (2003), pp. 517-532.

[8] A. Leal Duarte, Construction of acyclic matrices from spectral data, Lin. Alg. Appl. 113 (1989), pp. $173-182$.

[9] J.S. Maybee, D.D. Olesky, P. van den Driessche, and G. Wiener, Matrices, digraphs, and determinants, SIAM J. Matrix Anal. Appl. 10 (1989), pp. 500-519.

[10] P. van Moerbeke, The spectrum of Jacobi matrices, Invent. Math. 37 (1976), pp. 45-81. 